\title{
To evaluate and compare the Effectiveness of Sensorimotor Integration with that of Conventional Training for improving Balance and Gait in Stroke Hemiparesis: A Comparative Study
}

\author{
${ }^{1}$ Gaurav Agarwal, ${ }^{2}$ Anjali Jain
}

\begin{abstract}
Aim of the study: To evaluate and compare the effectiveness of sensorimotor integration with that of conventional training for improving balance and gait in stroke hemiparesis.
\end{abstract}

Materials and methods: The study design used for this research will be comparative study. Data will be taken from Mahatma Gandhi Hospital and Medical College, Jaipur and Mumbai.

Results: When both the groups were compared using unpaired t-test, sensorimotor group showed significant improvement in all outcome measures $(p<0.0001)$ except for MCTSIAB conditions 1 and 2 where the difference was not statistically significant.

Conclusion: Sensorimotor integration training is one of the novel treatment which can have a addictive effects along with the conventional training for balance.

Keywords: Balance and gait training, Sensorimotor integration.

How to cite this article: Agarwal G, Jain A. To evaluate and compare the Effectiveness of Sensorimotor Integration with that of Conventional Training for improving Balance and Gait in Stroke Hemiparesis: A Comparative Study. J Mahatma Gandhi Univ Med Sci Tech 2016;1(2):47-54.

Source of support: Nil

Conflict of interest: None

\section{INTRODUCTION}

Stroke is third common cause of death and also a major cause of long-term disability. Balance problem are thought to be common after stroke, and they have been implicated in the poor recovery of activities of daily living (ADL) and mobility and an increased risk of falls.

The specific causes of balance disorders in hemiparetic patients after stroke can be manifolded. ${ }^{1}$ Balance

\footnotetext{
${ }^{1,2}$ Assistant Professor

1,2Department of Physiotherapy, Mahatma Gandhi University of Medical Sciences and Technology, Mahatma Gandhi Physiotherapy College, Mahatma Gandhi Hospital, Jaipur Rajasthan, India

Corresponding Author: Anjali Jain, Assistant Professor Department of Physiotherapy, Mahatma Gandhi University of Medical Sciences and Technology, Mahatma Gandhi Physiotherapy College Mahatma Gandhi Hospital, Jaipur, Rajasthan, India
}

can be affected in various ways which include joint motion limitation, weakness, altered muscular tone, sensory deficits, anomalous postural reactions and cognitive problems. $^{2}$ Balance is a prerequisite for all functional activities and depends on the integrity of the central nervous system. Impaired balance and increased risk of falling toward the paretic side is found to be significantly correlated with locomotor function, functional abilities and length of staying inpatients rehabilitation facilities.

Balance impairment in patients with stroke hemiparesis is frequently related to deficits of central integration of afferent inputs (somatosensory, visual, and vestibular). Three sensory modalities are mainly involved in postural control: Somatosensory, visual, and vestibular afferents. Integration of information from these systems is crucial for adequate postural control. ${ }^{3}$ For instance, in the static standing position, healthy adults normally use somatosensory information which globally comes from the lower limbs (feet pressure receptors, ankle joint receptors and muscle proprioceptors) in order to build the main reference coordinates for balance. ${ }^{4,5}$ Under this condition, somatosensory afferents account for 70\% of the information required for postural control, while vestibular afferent accounts for $20 \%$ and visual input for $10 \% .^{6}$

Visual and vestibular inputs are likely to be more relevant sources of information when proprioceptive information is unreliable, for instance, during sway, ${ }^{6-9}$ When lower limb somatosensory information is inadequate (e.g., under a compliant surface support condition), other sensory systems are involved. This central integration of sensory inputs allows potential sensory conflicts generated by inadequate afferent information to be overcome.

For example, vestibular and somatosensory information is in conflict with visual information when a stationary train creates the illusion of movement as another train begins to move. Under these very critical conditions, the ability to analyze, compare and select the pertinent sensory information is very important in order to avoid falling. The existence of a general neural process 
aimed at resolving sensory conflicts and synthesizing from disparate sensory inputs and combining efferent and afferent information has already been suggested in literature. $^{10}$

Sensory information is regulated dynamically and modified by changes in environmental conditions. ${ }^{6}$ Despite the availability of multiple sources of sensory information, in a given situation, the central nervous system (CNS) gives priority to one system over another to control balance in the orthostatic position. ${ }^{11}$ The ability to choose and rely on the appropriate sensory input for each condition is called eweighting. ${ }^{12,13}$

When one is standing on an unstable surface, for instance, the CNS increases sensory weighting to vestibular and vestibular and visual information and decrease the dependence on surface somatosensory inputs for postural orientation. On the other hand, in darkness, balance control depends on somatosensory and vestibular feedback. Sensory reweighting is also important in the situation of sensory conflict that frequently occurs in daily activities; for example, when someone stands next to a bus in movement. In this situation, the visual system reports relative movement of the person in relation to an object, which conflicts with information from the somatosensory and vestibular systems. The CNS must reject visual information and use vestibular and somatosensory inputs. The ability to analyze, compare, and select the pertinent sensory information to prevent falls can be impaired in hemiparetic stroke patients. ${ }^{14}$

Abnormal interactions between the three sensory systems involved in balance could be the source of normal postural reactions. ${ }^{14-16}$ In situation of sensory conflict, a patient with stroke can inappropriately depend on one particular system over another. ${ }^{15}$ Laboratory measurements of sensory organization demonstrate that patients with chronic stroke perform worse in conditions of altered somatosensory information and visual deprivation or inaccurate visual input. ${ }^{14}$ Excessive reliance on visual input may be a learned compensatory response that occurs over time. ${ }^{14}$ Relying on a single system can lead to inappropriate adaptations and, hence, balance disturbances. Furthermore, sensory integration and reweighting can be impaired in patients with stroke, emphasizing visual input even when it provides inaccurate information. ${ }^{14-16}$

Several structures of the central nervous system seem to be involved in sensory integration such as the visual and vestibular cortex ${ }^{17,18}$ the posterior parietal cortex, ${ }^{19}$ the dorsoventral prefront al cortex ${ }_{1}^{19}$ the basal ganglia, ${ }^{20}$ the limbic system, ${ }^{21}$ the cerebellum, ${ }^{22}$ and the reticular system. $^{23}$
No general physiotherapy approach has been proven to be superior for promoting balance recovery from stroke.

More research is needed to identify effective and efficient interventions for improving functional balance and reducing the risk of falls among people living in the community after a stroke.

Although, a deficit of sensorimotor integration is now acknowledged as a major factor in balance disability after stroke, few studies ${ }^{2,24,25}$ have been carried out to propose remediation programs for rehabilitation of the anomalous processing of afferent sensory input in hemiplegic patients. A pilot study by Nicola et al have proved significant improvement in balance after rehabilitation of sensorimotor integration deficits in chronic stroke patients. But, there has been no research done to compare the efficacy of these new interventions with those of conventional physiotherapy for balance in chronic stroke patients. So, the main purpose of the study was to compare the efficacy of sensorimotor integration approach with that of conventional physiotherapy program for improving balance and gait in chronic stroke patients.

\section{AIM}

To evaluate and compare the effectiveness of sensorimotor integration with that of conventional training for improving balance and gait in stroke hemiparesis.

\section{OBJECTIVES}

- To assess the efficacy of conventional training on balance and gait in stroke hemiparesis.

- To assess the efficacy of sensorimotor integration on balance and gait in stroke hemiparesis.

- To compare the efficacy of conventional training and sensorimotor integration on balance and gait in stroke hemiparesis.

\section{MATERIALS AND METHODS}

Nature of study: The study design used for this research will be comparative study.

Research settings: Data will be taken from Mahatma Gandhi Hospital and Medical College Jaipur.

Selection criteria: An ethical committee approval was taken starting with the intervention.

A sample size of 30 subjects with stroke hemiparesis who met with the inclusion criteria was chosen from Mahatma Gandhi Hospital and Medical College (Jaipur). Their informed consent was taken. They were randomly divided into two groups: The control group and the experimental group. 
The control group was treated with conventional training and training group with sensorimotor integration.

\section{Inclusion criteria}

- First episode of stroke

- Stability of neurological severity

- Absence of any cognitive impairment according to mini mental scale

- Ability to stand and walk with/without an aid.

\section{Exclusion criteria}

- Perceptual and visual impairment

- Deficits of somatic sensation involving the paretic lower limb

- Vestibular disorders

- Paroxysmal vertigo

- Presence of neurological conditions such as neglect, hemianopia and controversies pushing syndrome

- Presence of orthopedic disease involving lower limbs.

\section{Materials required}

- Mat

- Ball

- Weight cuffs

- Foam surface (inclined wedge)

- Vestibular board

- Parallel bar

- Stool

- Mirror.

\section{Variables of study}

- Modified clinical test of sensory integration and balance (CTSIAB)

- 10 meters walk test

- Berg balance

- Dynamic gait index.

\section{Modified clinical test of sensory integration and balance (CTSIAB)}

The modified clinical test of sensory integration and balance is timed balance ${ }^{27-29}$ test that evaluate somatosensory, visual, and vestibular function for maintenance of upright posture. This test requires that patients maintain standing balance during a combination of three visual and two supports surface conditions. Tasks were performed with the eyes open and with the eyes closed. It included a hard, flat floor and an $8 \mathrm{~cm}$ section of density foam rubber that reduces the quality of the surface orientation input. During the test, subjects stood barefoot in the upright position with their arms alongside the body and their feet on the predesigned site. If the subjects activated any postural reaction, the test was stopped immediately and the number of seconds standing prior to the violation constituted the trial score.

The test was performed under four conditions:

1. Eyes open: Stable surface

2. Eyes open: Compliant surface

3. Eyes closed: Stable surface

4. Eyes closed: Compliant surface.

Five trials were carried out for each test condition. Each trial lasted 30 seconds. Total scores for each condition were the sums of the scores of each trial.

Maximum score for each test condition: $30 \times 5=150$.

\section{Ten Meters Walking Test}

This is a validated test used for quantitative analysis of gait. Patients were required to walk on a flat hard floor at their most comfortable pace for 10 meters using their usual assistive device and orthoses. Scoring was their walking speed.

\section{Berg Balance}

The Berg balance scale is a performance-based assessment tool, that is, used to evaluate standing balance during functional activities. The patients are scored on fourteen different tasks. Functional activities, such as reaching, bending, transferring, and standing, are evaluated on the test to evaluate balance.

Scores for each item can range from 0 (cannot perform) to 4 (normal performance).

Overall scores can range from 0 (severely impaired balance) to 56 (excellent balance).

- 41-56 = low fall risk

- $21-40=$ medium fall risk

- $0-20=$ high fall risk.

The Berg is considered as the gold standard assessment of balance with good intraratter reliability and good internal validity.

\section{Dynamic Gait Index}

It is a useful reliable and clinical tool for evaluating dynamic balance in ambulatory people with chronic stroke.

\section{Training Procedures}

All the subjects underwent 4 weeks of balance training program which consisted of 1 hour session thrice a week. The exercises for the conventional group were as follows: - Strengthening of trunk and lower limb muscles 
- Weight shifts in different positions, such as standing, kneeling, etc. Perturbations were given in different positions.

- Activities in standing included:

- Standing with feet close to each other

- Tandem standing

- Standing reach outs in all directions

- One leg standing

- Step up/down/forward/sideways

- Passing a ball behind

- Marching with head kept straight

- Standing with progression from wide to narrow BOS and then perturbations.

- Activities in walking included:

- Tandem walking

- Use of parallel bar (lateral walking)

- Walking over and around obstacles

- Picking up object while walking

- Carrying weights while walking

- Change in direction while walking.

The exercises for the sensorimotor integration group were as follows:

- Standing (progression from eyes open to closed and floor to mat):

- Weight shifts and reach outs:

- Tandem standing

- Tandem standing and reach outs

- One leg standing

- Standing with head turns to left and right as per commands and maintain balance with eyes closed.

- Standing on inclined wedge-progression with eyes closed and head turns:

- Step up from floor to mat, step up from floor to inclined wedge and back to floor

- Walking on mat (progression from eyes open to closed and head turns)

- Vestibular ball-sitting on ball with feet on floor and weight shifts/reach outs

- Progression with feet on mat and eyes closed and maintain balance in sitting and also head turns. With feet on mat and reach outs.

- Vestibular board-balancing on it in sagittal plane (progression from wide BOS to narrow, tandem)

- Use of scooter board-maintains standing balance while giving perturbations. Progression to eyes closed and then head turns.

\section{DATA ANALYSIS AND RESULTS}

There was significant improvement seen within both the groups on Berg balance $(\mathrm{p}<0.0001)$. Dynamic gait index $(\mathrm{p}$ $<0.0001), 10 \mathrm{~m}$ walk test $(\mathrm{p}=0.0001)$ and modified CTSIAB (all conditions) $(\mathrm{p}<0.0001)$ (Table 1 and Graphs 1 to 7$)$.
When both the groups were compared using unpaired t-test sensorimotor group showed significant improvement in all outcome measures $(p<0.0001)$ except for MCTSIAB conditions 1 and 2, and the difference was not statistically significant.

- Condition $1 ; \mathrm{p}>0.999$

- Condition 2; $\mathrm{p}=0.8562$

Table 1: Means and standard deviations of scores on outcome measures of conventional and experimental groups at baseline and 4 weeks

\begin{tabular}{lll}
\hline $\begin{array}{l}\text { Measures } \\
\text { Conventional }\end{array}$ & $\begin{array}{l}\text { Baseline (Pre) } \\
\text { Mean (SD) }\end{array}$ & $\begin{array}{l}\text { Post 4 weeks } \\
\text { Mean (SD) }\end{array}$ \\
\hline Berg balance & $38.66(8.27)$ & $45.46(6.34)$ \\
Dynamic gait index & $13.13(3.77)$ & $17.06(4.26)$ \\
10m walk test & $0.48(0.19)$ & $0.66(0.18)$ \\
MCTSIAB-1 & $144.6(6.39)$ & $150(0)$ \\
MCTSIAB-2 & $137.4(7.8)$ & $148.7(3.7)$ \\
MCTSIAB-3 & $87.33(15.79)$ & $93.73(13.22)$ \\
MCTSIAB-4 & $74.53(21.17)$ & $94.6(21.68)$ \\
Experimental & & \\
Berg Balance & $32.933(9.34)$ & $45.866(10.15)$ \\
Dynamic Gait Index & $13.06(3.283)$ & $19.86(2.85)$ \\
10m walk test & $0.46(0.15)$ & $0.79(0.16)$ \\
MCTSIAB-1 & $147.33(5.936)$ & $150(0)$ \\
MCTSIAB-2 & $138(13.2)$ & $150(0)$ \\
MCTSIAB-3 & $88.33(14.59)$ & $146(8.28)$ \\
MCTSIAB-4 & $54(12.42)$ & $142.66(14.37)$ \\
\hline & &
\end{tabular}

\section{DISCUSSION}

The present study shows that, following a specific training program based on weight transfer and balance exercises performed under different conditions of manipulation of sensory inputs, patients have achieved a significant improvement in their ability to maintain balance control. Gait ability, measured as walking speed, also showed a statistically significant improvement. These changes could not be ascribed to spontaneous recovery, because all the patients included in this study were at a chronic stage of illness. Thus, the improvement seen in the patients could be mainly ascribed to a change in sensory strategies used by patients in controlling their standing posture.

Balance is a complex process involving the reception and integration of sensory inputs and the planning and execution of movement to achieve a goal requiring upright posture. It is the ability to control the center of gravity (COG) over the base of support (BOS) in a given sensory environment. This biomechanical task of keeping COG over BOS is always accomplished within an environmental context which is detected by the sensory systems. 


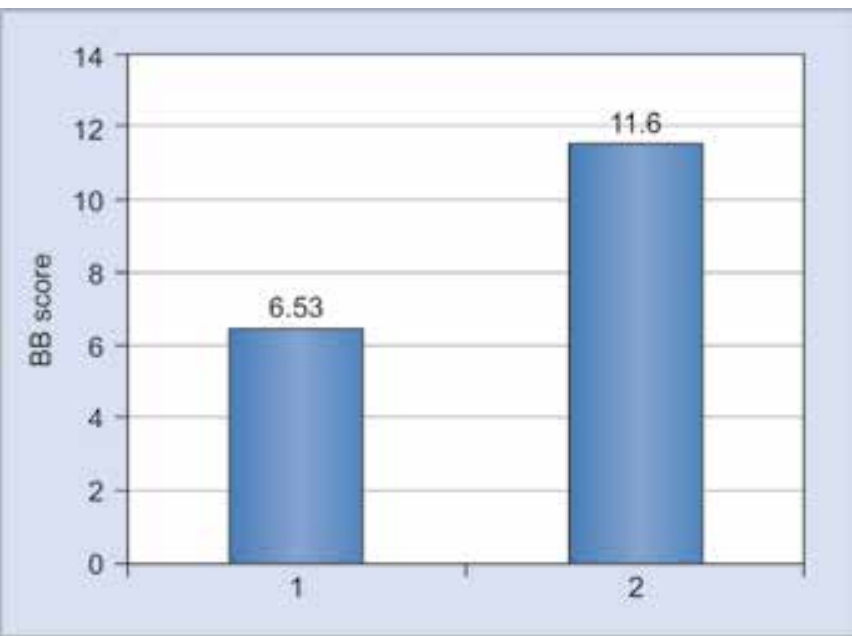

Graph 1: Improvement across conventional and experimental on Berg balance (BB) at 4 weeks

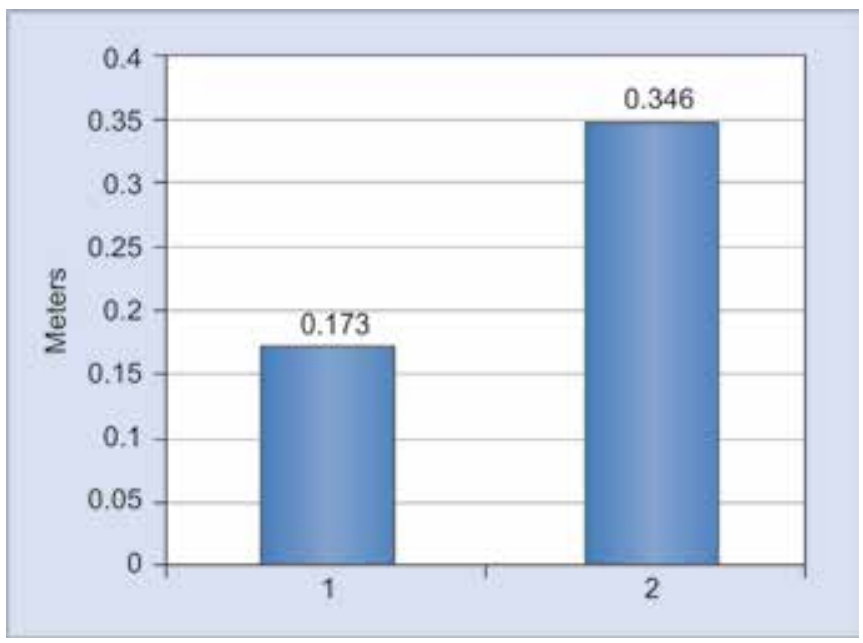

Graph 3: Improvement across conventional and experimental on $10 \mathrm{~m}$ walk test at 4 weeks

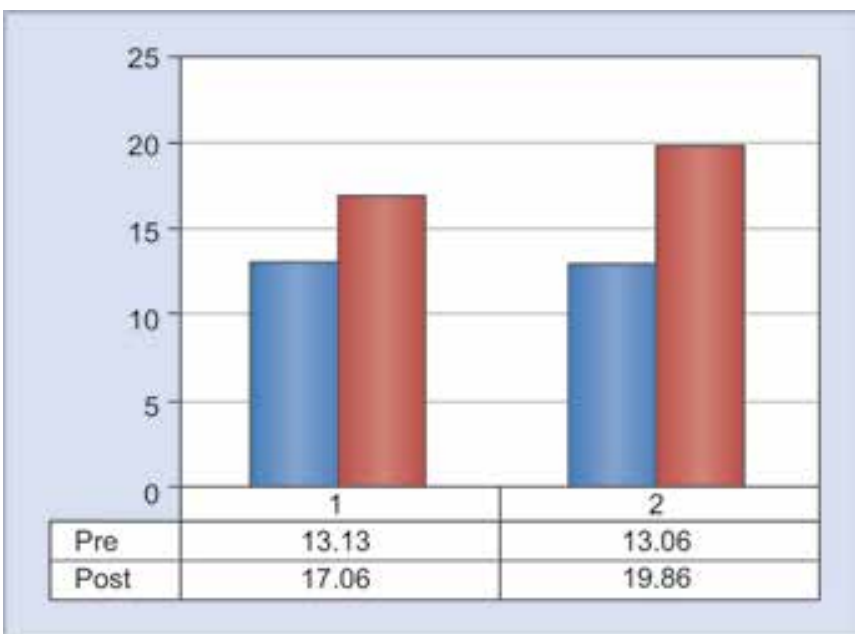

Graph 5: Dynamic gait index in conventional and experimental: Baseline and at 4 weeks

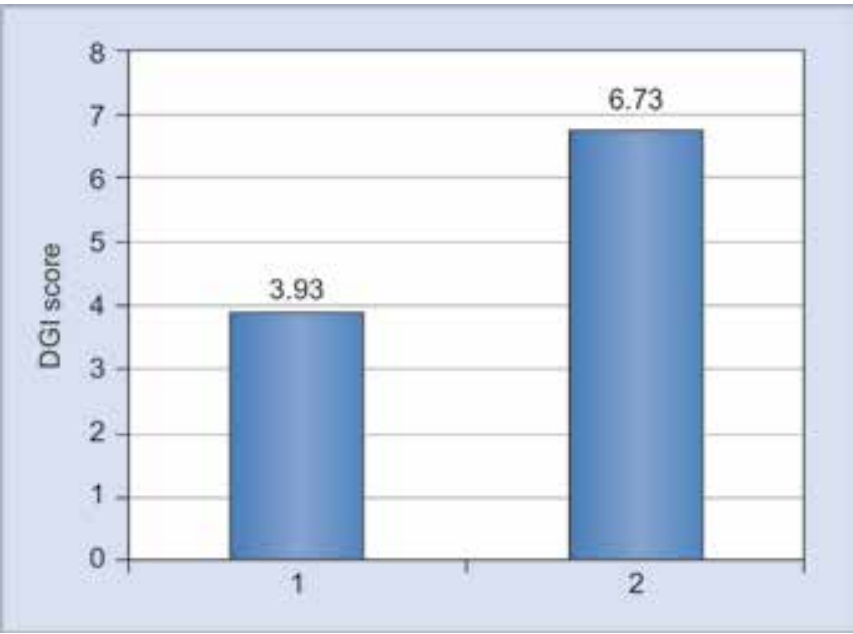

Graph 2: Improvement across conventional and experimental on Dynamic gait index (DGI) at 4 weeks

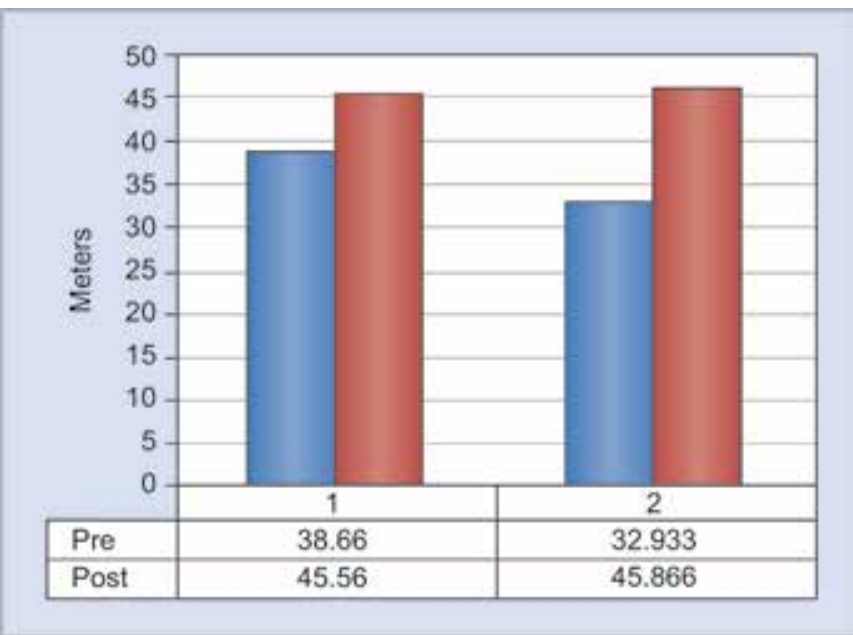

Graph 4: Berg balance in conventional and experimental: Baseline and at 4 weeks

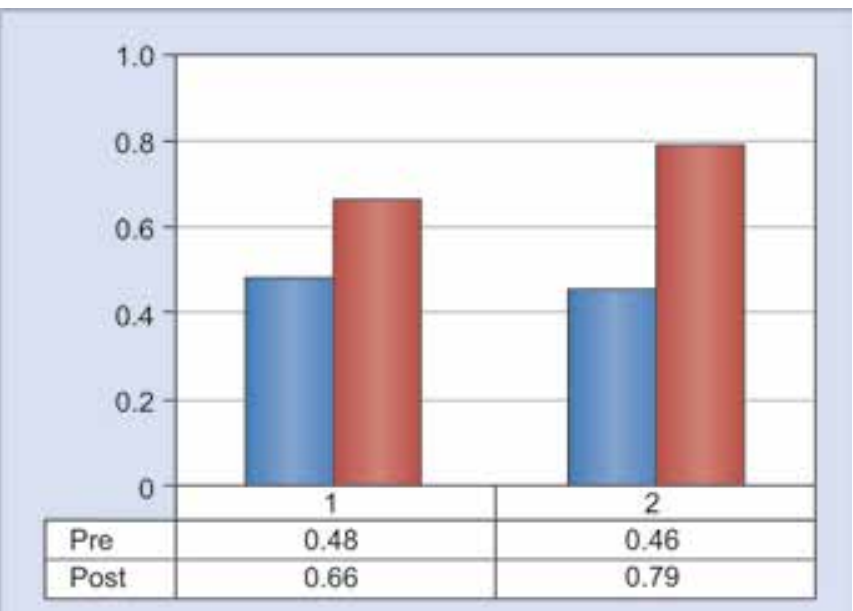

Graph 6: $10 \mathrm{~m}$ walk test in conventional and experimental groups: At baseline and after 4 weeks 


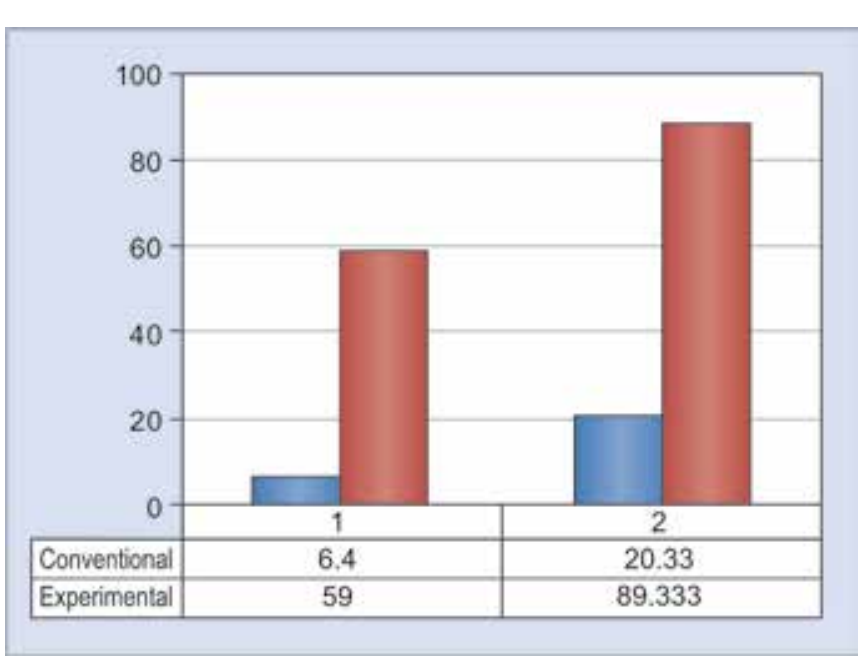

Graph 7: Improvement in modified CTSIAB (conditions 3 and 4) in conventional and experimental groups

The sensory environment is the set of conditions that exist, or are perceived to exist in the external world that may affect balance. Peripheral sensory receptors gather information about the environment, body positions and motion in relation to the environment, and the body segments positions and motions in relation to self. Central sensory structures process this information to perceive body orientation, position and motion and to determine the opportunities and limitation present in the environment.

Both the groups showed significant improvement on Berg balance and dynamic gait index $(\mathrm{p}<0.001)$. But sensorimotor group showed greater improvement than conventional group $(p<0.001)$.

Surface and visual conditions, however, may vary significantly and may be stable or unstable. Common unstable visual conditions are experienced on mass transit, in crowds or on a boat. Also, rapid head movement may render a stable visual environment unusable for postural cues, and darkness may preclude the use of vision.

As recently reported by Di Fabio and Badke ${ }^{30}$ unlike normal subjects, patients with stroke tend to rely upon visual rather than somatosensory inputs in order to maintain the standing posture. This observation was confirmed by pretreatment MCTSIAB data which showed that postural stability was markedly decreased in all the somatosensory conflicts (complaint surface) conditions, and in particular during the blind conditions.

Thus, following exercise training aimed at progressively inducing to use lower limb somatosensory inputs for controlling standing stability, patients are able to improve their ability to stand even in conditions in which somatosensory input has been altered in several ways. Therefore, patients who received sensorimotor training performed better than conventional group in conditions 3 and 4 of MCTSIAB.

Human balance requires appropriate integration of many sensory and motor systems. The brain uses three main senses-visual, vestibular and somatosensory to determine where one is in space and how one is moving in relation to the environment, visual information provides a reference of our movement in relationship to the environment. Somatosensory information is used in at least two different ways. It is from the feet and lower extremity is used to determine movement of the body in relation to the support system. This information from the neck and trunk is used in conjunction with information from vestibular system to determine if movement is of entire body or head alone.

It is very relevant from a functional point of view that similar improvement was also seen in walking speed. This could be explained by improvement in postural adjustment mechanisms. Movements of the legs are a source of disturbance of balance because they are involved in body support, and thus a displacement of the center of gravity is observed immediately before and after movement onset. The center of gravity shift occurs, during the initiation and course of gait. ${ }^{31}$ Control of the center of gravity shift toward a new position, compatible with equilibrium during movement, may be related both to anticipatory and responsive postural adjustments. ${ }^{31}$ It is worth noting that sensory input integration is very important for maintaining equilibrium, especially during conditions of perturbed balance, such as during walking performance. Hence, the sensorimotor group showed improved walking speed than the conventional group.

Bonan et $\mathrm{al}^{2,24}$ assessed twenty patients with chronic hemiplegia after stroke (more than 12 months from onset). Patients were randomized to two groups both of which underwent a 4-week balance rehabilitation program. ${ }^{32}$ Group I performed all the program exercises under vision deprivation while the same exercises were performed under free vision in group II. The results of this study showed that static and dynamic balance improved more after rehabilitation under visual deprivation than under free vision. They also recorded a significant improvement in gait ability after the training.

In the second study, by Bayouk et $\mathrm{al}^{25}$ sixteen patients with chronic hemiplegia after stroke (more than 12 months from onset) were randomized to an experimental and to a control group. The control group underwent an 8-week rehabilitation program aimed at improving balance, gait ability and movement coordination. These exercises were also performed in the experimental group but, in this case, the program also included exercises executed while the proprioception 
of the feet and ankles and/or vision was manipulated. As a whole, both groups received the same amount of therapy. As in the study by Bonan et $\mathrm{al}^{2,24}$ a significant improvement in static and dynamic balance was recorded after rehabilitation training. In contrast, an improvement in walking speed was observed not only in the experimental but also in the control group.

This specific effect of rehabilitation could be ascribed to the fact that both groups performed walking exercises according to their training program:

The results of this study extended previous results ${ }^{2,24}$ showing that a somatosensory integration training program can improve balance ability in patients with stroke and that this improvement is not transient but may persist for several days. As Perry et $\mathrm{al}^{26}$ described, hemiparetic patients with a mean walking speed of $0.26 \pm 0.11 \mathrm{~m} / \mathrm{s}$ can be classified as those who are able to use walking for all household activities but unable to enter and leave their homes independently. On the other hand, patients with a walking speed of $0.4 \pm 0.18 \mathrm{~m} / \mathrm{s}$ are classified as being capable of entering and leaving their homes independently. ${ }^{33-37}$

Although the mechanism underlying the improvement in gait performance after a somatosensory integration training program are still subject to debate, ${ }^{25}$ the results of the present study suggest that a balance rehabilitation program may lead to an improvement in postural control mechanism and consequently in gait performance.

\section{LIMITATION}

- The small sample size. Further studies with a larger subject population are needed to further validate the findings.

\section{CONCLUSION}

These results showed that the addition of a multisensory training component to the regular exercise program was required to obtain a significant improvement in standing balance of stroke subjects. In the absence of sensory training, very limited changes were observed for both static and dynamic balance tasks. Thus, it could be explained that sensorimotor integration training is one of the novel treatment which can have a addictive effects along with the conventional training for balance.

\section{REFERENCES}

1. Tyson SF, Hanley M, Chillala J, et al. Balance disability after stroke. Phys Ther 2006;86:30-38.

2. Bonan IV, Colle FM, Guichard JP, et al. Reliance on visual information after stroke. Part 1: Balance on dynamic posturography. Arch Phys Med Rehabil 2004;85:268-273.
3. Horak FB. Clinical assessment of balance disorders. Gait Posture 1997;6:76-84.

4. Maurer C, Mergner T, Peterka RJ. Multisensory control of human upright stance. Exp Brain Res 2006;171:231-250.

5. Mergner T, Schweigart G, Maurer C, Blumle A. Human postural responses to motion of real and virtual visual enviroments under different support base conditions. Exp Brain Res 2005;167:535-556.

6. Peterka JR. Sensorimotor integration in human postural control. J Neurophysiolol 2002;88(3):1097-1118.

7. Fitzpatrick RC, McCloskey DJ. Proprioceptive, visual and vestibular threshold for the perception of sway during standing in humans. J Physiol 1994;478(Pt I):173-186.

8. Peterka RJ, Loughlin PJ. Dynamic regulation of sensorimotor integration in human postural control. J Neurophysiol 2004;91(1):410-423.

9. Oie K, Kiemel T, Jeka JJ. Multisensory fusion: Simultaneous re-weighting of vision and touch for the control of human posture. Brain Res Cogn Brain Res 2002;14(1):164-176.

10. Merfeld DM, Zupan L, Peterka RJ. Human use internal models to estimate gravity and linear acceleration. Nature 1999;398:615-618.

11. Forssberg H, Nashner LM. Ontogenetic development of postural control in man: adaptation to altered support and visual conditions during stance. J Neurosci 1982;2(5):545-552.

12. Shumway-Cook A, Woollacott MH. Motor control: theory and practical applications. 2nd ed. Philadelphia(PA): Lippincott, Williams and Wilkins; 2001.

13. Horak FB. Postural orientation and equilibrium. What do we need to know about neural control of balance to prevent falls? Age Ageing 2006;35 Suppl 2:ii7-ii11.

14. Bonan IV, Colle FM, Guichard JP, Viacut E, Eisenfisz M, Tran Ba Huy P, Yelnik AP. Reliance on visual information after stroke. Part I: Balance on dynamic post urography. Arch Phys Med Rehabil 2004;85(2):268-273.

15. Nashner LM, Black FO, Wall C IIIrd. Adaptation to altered support and visual conditions during stance: Patience with vestibular deficits. J Neurosci 1982;2(5):536-544.

16. Nashner LM, Shumway-Cook A, Marin O. Stance posture control in select groups of children with cerebral palsy: deficits in sensory organisation and muscular coordination. Exp Brain Res 1983;49(3):393-409.

17. Bonan IV, Leman MC, Legargasson JF, et al. Evolution of subjective visual vertical perturbation after stroke. Neurorehabil Neural Repair 2006;20:484-491.

18. Brandt T, Glasauer S, Stephan T, et al. Visual-vestibular and visuovisual cortical interaction: new insights from $\mathrm{fMRI}$ and PET. Ann NY Acad Sci 2002;956:230-241.

19. Fink GR, Marshall JC, Halligan PW, et al. The neural consequences of conflict between intention and the senses. Brain 1999;122:497-512.

20. Connor NP, Abbs JH. Sensorimotor contribution of the basal ganglia: recent advances. Phys Ther 1999;70:864-872.

21. Kohl RL. Sensory conflict theory of space motion sickness: an anatomical location for the neuroconflicts. A viat Space Environ Med 1983;54:464-465.

22. Parson LM, Bower JM, Gao JH, et al. Lateral cerebellar hemispheres actively support acquisition and discrimination rather than motor control. Learn Mem 1997;4:49-62.

23. Precht W. Vestibular mechanisms. Annu Rev Neurosci 1979;2:265-289. 
24. Bonan IV, Yelnik AP, Colle FM, et al. Reliance on visual information after stroke. Part II: Effectiveness of a balance rehabilitation program with visual cue deprivation after stroke: a randomized controlled trial. Arch Phys Med Rehabil 2004;85:274-278.

25. Bayouk JF, Bouncher JP, Leroux A. Balance training following stroke: effects of task-oriented exercises with and without altered sensory input. Int J Rehabil Res 2006;29:51-59.

26. Maeda A, Yuasa T, Nakamura K, et al. Physical performance tests after stroke: reliability and validity. Am J Phys Med Rehabil 2000;79:519-525.

27. Di Fabio RP, Badke MB. Relationship of sensory organisation to balance function in patients with hemiplegia. Phys Ther 1990;70:542-548.

28. Maeda A, Yuasa T, Nakamura K, et al. Physical performance tests after stroke: reliability and validity. Am J Phys Med Rehabil 2000;79:519-525.

29. Cohen H, Heaton LG, Congdon SL, Jenkins HA. Changes in Sensory Organisation Test Scores with age. Age Aging 1996;25:39-44.
30. Di Fabio RP, Badke MB. Stance duration under sensory conflicts conditions in patients with hemiplegia. Arch Phys Med Rehabil 1991;72:292-295.

31. Massion J, Woollacott $\mathrm{MH}$, editors. Clinical disorders of balance posture and gait, 1st edn. Arnold, London.

32. Wood-Dauphinee S, Berg K, Bravo G, Williams JI. The balance scale: responding to clinically meaningful changes. Canadian J of Rehabil 1997;10:35-50.

33. Berg K, Wood-Dauphinee S, Williams JI. The balance scale: reliability assessment for elderly residents and patients with acute stoke. Scand J Rehab Med 1995;27:27-36.

34. Berg K, Maki B, Williams JI, Holiday P, Wood-Dauphinee S. A comparison of clinical and laboratory measures of postural balance in an elderly population. Arch Phys med Rehabil 1992;73:1073-1083.

35. Berg K, Wood-Dauphinee S, Williams JI, Maki B. Measuring balance in the elderly: validation of an instrument. Can J Pub Health 1992 Jul/Aug; Supplement 2:7-11.

36. Berg K, Wood-Dauphinee S, Williams JI, Gayton D. Measuring balance in the elderly: preliminary development of an instrument. Physiotherapy Canada 1989;41:304-311.

37. Shumway-Cook A, Wollacott M Motor control: theory and practical applications. Baltimore: Williams an Wilkins; 1995. 